\title{
Effect of Temperature on Fatty Acid Metabolism in Dissociated Cell Cultures of Developing Brain
}

\author{
Ephraim Yavin and John H. Menkes ${ }^{[25]}$ \\ Division of Pediatric Neurology, University of California at Los Angeles, and the Brentwood Veterans Administration Hospital, \\ Los Angeles, California, USA
}

\section{Extract}

The effect of hypothermia on the incorporation and metabolism of essential fatty acids (linoleic and linolenic acids) by dissociated cell cultures of developing brain was examined. When the ambient temperature was reduced to $30^{\circ}$, chain elongation of linolenic acid (18:3) to 20:3, and elongation of 20:5 to 22:5 was slowed. Desaturation of $20: 3$ to $20: 4$ and 20:5 remained unaffected even at $22^{\circ}$. Incorporation of $\left(1-{ }^{14} \mathrm{C}\right)$ linolenic acid into the fraction containing ethanolamine phosphoglycerides and ethanolamine plasmalogens (EPG) was significantly reduced by hypothermia, whereas labeling of choline phosphoglycerides (CPG) was greater at $30^{\circ}$ and $22^{\circ}$ than at $37^{\circ}$, and fell only slightly at $15^{\circ}$. These observations suggest that EPG labeling depends on the availability of the higher polyunsaturated fatty acids (22:5 and 22:6), whereas CPG labeling is nonspecific. Because ethanolamine plasmalogens are major myelin constituents, the importance of hypothermia in inducing a deficiency of the higher polyunsaturated fatty acids within the developing nervous system, and thus interfering with the biosynthesis of EPG and indirectly with membrane deposition, needs to be clarified.

\section{Speculation}

These studies suggest that hypothermia may induce a deficiency of the higher polyunsaturated fatty acids in the developing nervous system, which, in turn, could induce a defect in the formation of cell membranes. Increases in the concentration of essential fatty acids for formulas intended for low birth weight infants might compensate for this relative deficiency.

\section{Introduction}

There is considerable evidence that the survival rate of premature infants is adversely affected by an environment that stresses their thermoregulatory system $[2-5,12]$. The metabolic derangements that occur within the nervous system of infants in the course of acute or prolonged hypothermia have not been fully explored, but it appears likely that permanent alterations may occur under such circumstances. To our knowledge there are no long term follow-up studies of premature infants which correlate the incidence of subsequent neurologic handicaps with periods of neonatal hypothermia. The neurologic picture of older infants suffering from exposure to cold, sufficiently prolonged to lower body temperature to $32^{\circ}$ or less, includes apathy and coarse tremors which may progress to generalized convulsions. The latter may be the result of a primary effect on the central nervous system, or be induced by hypoglycemia [6].

In newborn rats reduction of the ambient temperature is accompanied by reduced incorporation of 

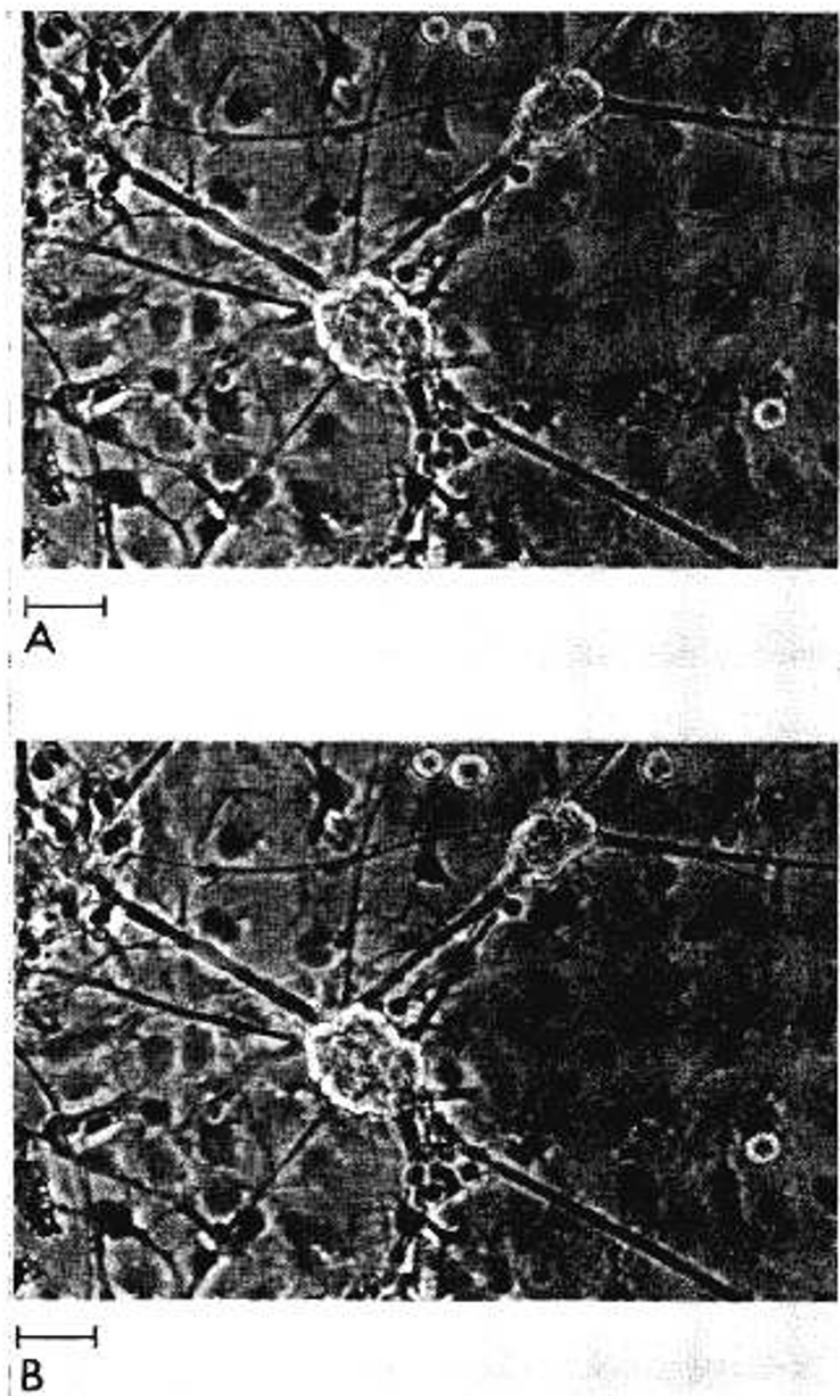

Fig. 1. Cultured dissociated cells from 14-17-day rat embryo cerebral hemisphere, 11 days in vitro. Bar denotes $20 \mu \mathrm{m}$.

leucine into brain proteins [8]. This effect is apparently the direct result of lowered body temperature, rather than being caused by energy depletion which accompanies thermogenesis.

The availability of cultured dissociated cells from fetal brain has prompted us to utilize this system in studies of lipid biosynthesis and fatty acid metabolism, two processes vital for the formation and stability of membranes in the developing nervous system.

Mechanically or enzymatically dissociated brain cells reassociate to form well organized aggregates in the course of culture. These acquire some of the morphologic and biochemical characteristics of the differentiated original tissue $[1,9,10,13,14]$. Three cell types can usually be distinguished: neuron-like cells with little mitotic activity; small, dense, glia-like cells; and large, clear, polygonal ependyma-like cells [10, 13, 14] (Fig. 1).

Under these conditions cellular lipids are synthesized from precursors in the culture medium, and dissociated brain cells take up exogenous free fatty acids and glycerol rapidly and utilize them in the synthesis of cellular triacylglycerols (TG) and phospholipids. The relative proportions of free fatty acids entering these two lipid fractions depend in part on the age of the brain cell cultures. In the early stages of culture when outgrowth of cell processes is active, fatty acids are preferentially incorporated into phospholipids, whereas later, when cell division has slowed down, incorporation of fatty acids into TG is increased [15]. These observations are consistent with the concept that, in brain cells, TG serve as an intracellular reservoir for excess free fatty acids.

Within the phospholipid fraction, CPG and EPG are the major labeled species. As separated in our laboratory, the EPG fraction contains both ethanolamine phosphoglycerides and phosphatidal ethanolamines (ethanolamine plasmalogens). With increasing chain length and increasing unsaturation of the labeled fatty acid precursors, the proportion of radioactivity incorporated into EPG to that entering CPG increases progressively. In the course of their incorporation into cellular lipids, fatty acids undergo chain elongation and desaturation. In previous studies we demonstrated that when linolenic acid (18:3) was used as precursor, the relative amount of label entering phospholipid was greatest. The EPG fraction was labeled more slowly than the CPG fraction, and the entry of radioactivity into EPG paralleled the conversion of 18:3 to docosapentaenoic acid (22:5), and docosahexaenoic acid (22:6), two major EPG fatty acids [16].

As preliminary observations suggested that biosynthesis of polyunsaturated fatty acids from the two essential fatty acids in the medium (18:3) and linoleic acid (18:2), was highly sensitive to the ambient temperature of the cultures [17], further studies to elucidate this phenomenon were in order.

\section{Materials and Methods}

\section{Preparation and Culture of Dissociated Cerebral Cells}

Monolayered cultures were prepared from cerebral hemispheres of 17-day-old rat embryos as described in 
detail [14]. Dissociated cells prepared by trypsinization were allowed to grow in $60-\mathrm{mm}$ Petri dishes at $37^{\circ}$ in an atmosphere of $95 \%$ air and $5 \% \mathrm{CO}_{2}$. The usual inoculum was between 0.5 and $1.0 \times 10^{6}$ cells/Petri dish. The nutrient medium consisted of Eagle's minimal essential medium [18] to which $20 \%$ of heattreated fetal calf serum [19], 0.6\% glucose, and antibiotics had been added. The $\mathrm{pH}$ of the medium was maintained between 7.0 and 7.4. Depending on the acidity of the culture, the medium was changed every 2-3 days. The amount of cellular DNA harvested from each Petri dish ranged from $20-40 \mu \mathrm{g}$.

\section{Incubation of Cultures}

Metabolic studies were conducted after cultures had reached confluency. After the nutrient medium was removed and the cells were washed once with serum-free Medium 199 [18], the experimental medium, consisting of 2-3 ml Medium 199 augmented with $0.6 \%$ glucose and containing the labeled fatty acid, was added. Cultures were examined daily by phase microscopy; no morphologic changes were observed in the course of the incubation period. Ambient temperatures for the metabolic studies were as specified.

\section{Substrates}

$\left(1-{ }^{14} \mathrm{C}\right)$ Linoleic acid $(57 \mathrm{mCi} / \mathrm{mmol})$ and $\left(1-{ }^{14} \mathrm{C}\right)$ linolenic acid $(52.4 \mathrm{mCi} / \mathrm{mmol})$ [20] were complexed to fatty acid-free albumin as described previously [15]. In this form fatty acids were stable for several months when stored at $-20^{\circ}$. Purity of substrates was verified both by thin layer chromatography (TLC) and gasliquid chromatography (GLG).

\section{Extraction and Fractionation of Cellular Lipids}

Most of the procedures have been described elsewhere [15]. In essence, cellular lipids were extracted with chloroform-methanol $(1 / 2, \mathrm{v} / \mathrm{v})$. To the organic extract chloroform and water were added to give a final ratio of chloroform-methanol-water of $8 / 4 / 3$, and the ensuing aqueous phase was removed. The lipid extract was placed on TLC plates ( 10 by $20 \mathrm{~cm}$ ) coated with silica gel $\mathrm{G}$ [21] and developed either in chloroform-methanol-acetic acid-water $(25 / 15 / 3 / 2, \mathrm{v} / \mathrm{v})$ or chloroform-methanol-7 $\mathrm{N}$ ammonia $(65 / 35 / 5, \mathrm{v} / \mathrm{v})$. Areas corresponding to the various phospholipid fractions were eluted with methanol. Separation of the neutral lipids was performed on silica gel G TLC plates in one direction, successively with dichloroeth- ane and a mixture of petroleum ether (boiling range $\left.30-60^{\circ}\right)$-diethyl ether-formic acid $(50 / 50 / 1.5, \mathrm{v} / \mathrm{v})$.

\section{Gas-Liquid Chromatography}

Gas-liquid chromatography was performed as described previously [15]. In essence, the purified lipid fractions isolated by means of TLC were methylated in $0.5 \mathrm{~N} \mathrm{HCl}$ in methanol, and the resultant fatty acid methyl esters were analyzed on a Hewlett-Packard (402) gas chromatograph equipped with a radioactivity monitor. Supelcoport columns coated with $10 \%$ SP-216-PS [22] were run at $200^{\circ}$ under 75 pounds argon pressure.

\section{Results}

\section{Incorporation of $\left(1-{ }^{14} C\right)$ Linoleic Acid into Cellular Lipids}

When cultures were incubated at $37^{\circ}$ for $16 \mathrm{hr}$ with labeled linoleic acid, radioactivity entered the CPG, EPG, and TG fractions preferentially. Upon lowering the incubation temperature, the total amount of radioactivity incorporated into cellular lipids showed a steady drop (Table I). Although the amount of radioactivity entering the EPG and TG fractions fell off markedly, CPG and serine phosphoglyceride labeling at $30^{\circ}$ and $22^{\circ}$ was greater than at $37^{\circ}$. As a consequence, the ratio of radioactivity in EPG to that in CPG fell progressively with lowering of the incubation temperature.

Among neutral lipids, TG labeling was reduced the most. By contrast, the amount of radioactivity in the fraction containing monoacylglycerols, and free, cellbound fatty acids was greater at $30^{\circ}$ than at $37^{\circ}$, and was about the same at $15^{\circ}$ as at $37^{\circ}$.

\section{Linoleic Acid Metabolism}

With reduction in the incubation temperature, conversion of $18: 2$ to other $\omega 6$ fatty acids dropped steadily, so that when cells were incubated at $15^{\circ}$ essentially all of the label remained in the form of $18: 2$ (Table II). Figure 2 shows the distribution of radioactivity in fatty acids isolated from cellular EPG and CPG. Since at $37^{\circ}$ and at $30^{\circ} 20: 3$ and $20: 4$ constitute a significant proportion of labeled EPG fatty acids, their failure to be synthesized is, in part, responsible for the reduced formation of EPG. By contrast, even at $37^{\circ}$ very little radioactivity enters $20: 3$ and 20:4 of the CPG fraction. With reduction in the incubation temperature 
Table I. Incorporation of $\left(1-{ }^{-14} \mathrm{C}\right)$ linoleic acid into lipid classes of cultured brain cells at various temperatures:

\begin{tabular}{|c|c|c|c|c|}
\hline \multirow{2}{*}{ Lipid fraction } & \multicolumn{4}{|c|}{ Temperature } \\
\hline & $15^{\circ}$ & $22^{\circ}$ & $30^{\circ}$ & $37^{\circ}$ \\
\hline \multirow[t]{2}{*}{ Triacylglycerol } & 30 & 50 & 210 & 747 \\
\hline & \pm 4 & \pm 9 & \pm 14 & \pm 92 \\
\hline \multirow[t]{2}{*}{ Diacylglycerol } & 38 & 73 & 114 & 148 \\
\hline & \pm 4 & \pm 11 & \pm 5 & \pm 38 \\
\hline \multirow[t]{2}{*}{ Free fatty acid ${ }^{2}$} & 54 & 56 & 200 & 78 \\
\hline & \pm 12 & \pm 4 & \pm 14 & \pm 16 \\
\hline \multirow[t]{2}{*}{ Cholesterol ester } & 8 & 10 & 36 & 81 \\
\hline & \pm 2 & \pm 2 & \pm 8 & \pm 13 \\
\hline \multirow[t]{2}{*}{ CPG } & 2,034 & 4,126 & 4,613 & 3,198 \\
\hline & \pm 281 & \pm 263 & \pm 145 & \pm 180 \\
\hline \multirow[t]{2}{*}{$\mathrm{SPG}^{3}$} & 101 & 236 & 622 & 155 \\
\hline & \pm 10 & \pm 58 & \pm 130 & \pm 72 \\
\hline \multirow[t]{2}{*}{$\mathrm{EPG}^{4}$} & 233 & 1,223 & 2,297 & 3,209 \\
\hline & \pm 108 & \pm 192 & \pm 166 & \pm 418 \\
\hline \multirow[t]{2}{*}{ Total incorporation } & 2,585 & 5,913 & 7,813 & 8,821 \\
\hline & \pm 447 & \pm 446 & \pm 436 & \pm 286 \\
\hline $\mathrm{EPG} / \mathrm{CPG}$ & 0.11 & 0.29 & 0.49 & 1.00 \\
\hline
\end{tabular}

${ }^{1}$ Cultured dissociated brain cells, 13 days in vitro, were incubated for $16 \mathrm{hr}$ with $\left(1-{ }^{14} \mathrm{C}\right)$ linoleic acid $(2.0 \mu \mathrm{M})\left(0.73 \times 10^{6}\right.$ $\mathrm{dpm} /$ culture) at various temperatures. The radioactive medium was removed and cells washed twice with albumin-saline solution. Cellular lipids were extracted and fractionated as described in Methods. Radioactivity expressed as disintegrations per minute per microgram of DNA represents mean \pm SD of three to four cultures. CPG : choline phosphoglycerides; SPG: serine phosphoglycerides; EPG: ethanolamine phosphoglycerides and plasmalogens.

${ }^{2}$ Also contains monoglyceride.

${ }^{3}$ Also contains inositol phosphoglycerides.

4 Also contains cardiolipin.

Table II. Distribution of $\left(1-{ }^{14} \mathrm{C}\right)$ linoleic acid and its metabolites in total cell fatty acids at various temperatures ${ }^{1}$

\begin{tabular}{|c|c|c|c|c|}
\hline \multirow{2}{*}{$\begin{array}{c}(\omega 6) \\
\text { Fatty acids }\end{array}$} & \multicolumn{4}{|c|}{ Temperature } \\
\hline & $15^{\circ}$ & $22^{\circ}$ & $30^{\circ}$ & $37^{\circ}$ \\
\hline $18: 2$ & 98.1 & $90.0 \pm 2.9$ & $70.4 \pm 1.8$ & $44.0 \pm 4.3$ \\
\hline $20: 2^{2}$ & 0 & 0 & 0 & $1.5 \pm 0.6$ \\
\hline $20: 3$ & 1.8 & $5.2 \pm 1.4$ & $9.8 \pm 0.9$ & $11.4 \pm 2.6$ \\
\hline $20: 4$ & 0 & $4.6 \pm 1.6$ & $19.5+2.3$ & $40.9 \pm 3.5$ \\
\hline $16: 0-18: 0$ & 0 & $\overline{0}$ & 0 & $2.2 \pm 0.3$ \\
\hline
\end{tabular}

${ }_{1}$ Experimental conditions are the same as described for Table I. Samples of the whole lipid extract were transmethylated and analyzed by gas-liquid chromatography as described in Methods. Values represent percentage of distribution of radioactivity \pm sD of three to four cultures.

${ }^{2}$ Tentatively identified.

there is a significant increase in the amount of labeled 18:2 in CPG, so that the increased CPG labeling at $22^{\circ}$ and $30^{\circ}$ is entirely due to an increased acylation of $18: 2$.

\section{Incorporation of $\left(1^{-14} G\right)$ Linolenic Acid into Cellular Lipids}

Reducing the incubation temperature resulted in a diminution of the linolenic acid incorporation into total cellular lipids. As was observed for linoleic acid, linolenic acid incorporation into EPG and TG was very sensitive to a reduced ambient temperature, whereas incorporation into CPG and serine phosphoglycerides, and into the fraction containing free fatty acids and monoacylglycerols was actually greater at $30^{\circ}$ than at $37^{\circ}$ (Table III).

Conversion of $18: 3$ to $22: 5$ and $22: 6$ was reduced markedly at $30^{\circ}$, and completely abolished at $22^{\circ}$, whereas desaturation of $20: 3$ to $20: 4$ and further desaturation of $20: 4$ to $20: 5$ was still active at $30^{\circ}$ and at $22^{\circ}$, and only became significantly reduced at $15^{\circ}$ (Table IV).

As can be seen from Figure 3, reduced incorporation of labeled linolenic acid into the EPG fraction when incubation temperatures were lowered was entirely because of the inadequate conversion of 18:3 to higher $\omega 3$ polyunsaturated fatty acids. About onehalf of the reduction in labeling of EPG 20:4, 20:5, 22:5, and 22:6 at $30^{\circ}$ can be accounted for by an increase in CPG labeling and by an increase in the con-

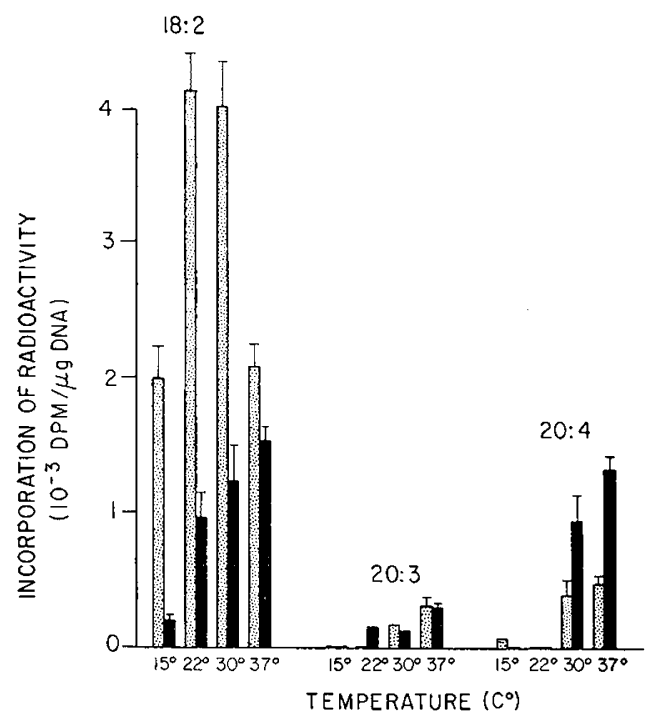

Fig. 2. Distribution of radioactivity from $\left(1^{14} \mathrm{C}\right)$ linoleic acid in fatty acid esters of choline phosphoglycerides and ethanolamine phosphoglycerides and plasmalogens (EPG). Incubation conditions were the same as described for Table I. Fatty acid methyl esters of CPG and EPG were analyzed by gas-liquid chromatography as described in Methods. Values expressed as disintegrations per minute per microgram of cellular DNA represent mean \pm SD of two analyses of three cultures. CPG: stippled bars; EPG: solid bars. 
Table III. Incorporation of $\left(1-{ }^{14} \mathrm{C}\right)$-linolenic acid into lipid classes of cultured brain cells at various temperatures ${ }^{1}$

\begin{tabular}{|c|c|c|c|c|}
\hline \multirow{2}{*}{ Lipid fraction } & \multicolumn{4}{|c|}{ Temperature } \\
\hline & $15^{\circ}$ & $22^{\circ}$ & $30^{\circ}$ & $37^{\circ}$ \\
\hline \multirow[t]{2}{*}{ Triacylglycerol } & 49 & 61 & 180 & 602 \\
\hline & \pm 3 & \pm 9 & \pm 37 & \pm 54 \\
\hline \multirow[t]{2}{*}{ Diacylglycerol } & 87 & 89 & 139 & 43 \\
\hline & \pm 11 & \pm 10 & \pm 20 & \pm 6 \\
\hline \multirow[t]{2}{*}{ Free fatty acid ${ }^{2}$} & 124 & 72 & 232 & 131 \\
\hline & \pm 9 & \pm 4 & \pm 107 & \pm 17 \\
\hline \multirow[t]{2}{*}{ Cholesterol ester } & 13 & 22 & 75 & 80 \\
\hline & \pm 2 & +4 & \pm 3 & \pm 30 \\
\hline \multirow[t]{2}{*}{ CPG } & 1,809 & 2,613 & 3,046 & 2,598 \\
\hline & \pm 145 & \pm 298 & \pm 137 & \pm 439 \\
\hline \multirow[t]{2}{*}{$\mathrm{SPG}^{3}$} & 236 & 293 & 819 & 548 \\
\hline & \pm 26 & \pm 31 & \pm 94 & \pm 187 \\
\hline \multirow[t]{2}{*}{$\mathrm{EPG}^{4}$} & 271 & 836 & 1,497 & 2,665 \\
\hline & \pm 61 & \pm 92 & \pm 281 & \pm 255 \\
\hline \multirow[t]{2}{*}{ Total incorporation } & 2,625 & 4,000 & 6,062 & 6,890 \\
\hline & \pm 206 & \pm 402 & \pm 247 & \pm 279 \\
\hline EPG/CPG & 0.14 & 0.31 & 0.49 & 1.02 \\
\hline
\end{tabular}

${ }_{1}^{1}$ Experimental conditions are described in Table I, except that the cells were incubated with $(1-14 \mathrm{C})$ Iinolenic acid $(2.3$ $\mu \mathrm{M})\left(0.81 \times 10^{6} \mathrm{dpm} /\right.$ culture $)$. Radioactivity expressed as disintegrations per minute per microgram of DNA represents mean \pm SD of three to four cultures. CPG: choline phosphoglycerides; SPG : serine phosphoglycerides; EPG : e thanolamine phosphoglycerides and plasmalogens.

${ }^{2}$ Also contains monoglycerides.

${ }^{3}$ Also contains inositol phosphoglycerides.

4 Also contains cardiolipin.

Table IV. Distribution of $(1-14 \mathrm{C})$ linolenic acid and its metabolites in total cell fatty acids at various temperatures ${ }^{1}$

\begin{tabular}{lcrrr}
\hline \multirow{2}{*}{$\begin{array}{c}(\omega 3) \\
\text { Fatty acids }\end{array}$} & \multicolumn{5}{c}{ Temperature } \\
\cline { 2 - 5 } & $15^{\circ}$ & \multicolumn{2}{c}{$22^{\circ}$} & \multicolumn{1}{c}{$30^{\circ}$} \\
\hline $18: 3$ & $96.0 \pm 1.2$ & $61.3 \pm 2.6$ & $35.1 \pm 2.6$ & $18.2 \pm 1.7$ \\
$20: 3$ & 0 & $5.7 \pm 0.9$ & $9.0 \pm 0.9$ & $13.3 \pm 1.1$ \\
$20: 4^{2}$ & $1.4 \pm 0.6$ & $10.5 \pm 1.2$ & $13.9 \pm 1.0$ & $18.6 \pm 2.2$ \\
$20: 5$ & $2.5 \pm 0.6$ & $22.3 \pm 3.6$ & $36.0 \pm 3.6$ & $29.3 \pm 3.1$ \\
$22: 5$ & 0 & 0 & $5.8 \pm 4.8$ & $13.9 \pm 1.0$ \\
$22: 6$ & 0 & 0 & 0 & $2.4 \pm 0.6$ \\
$16: 0-18: 0$ & 0 & 0 & 0 & $4.2 \pm 0.3$ \\
\hline
\end{tabular}

${ }_{1}$ Experimental conditions are the same as described in Table $\mathrm{I}$ and II except that cells were incubated with $(1-14 \mathrm{C})$ linolenic acid $(2.3 \mu \mathrm{M})\left(0.81 \times 10^{6} \mathrm{dpm} /\right.$ culture). Values represent percentage of distribution of radioactivity $\pm \mathrm{sD}$ of three to four cultures.

2 Tentatively identified.

tent of labeled 18:3 in EPG. Furthermore, the increase in CPG labeling at $30^{\circ}$ and $22^{\circ}$ was completely accounted for by increased incorporation of labeled 18:3 into CPG. With lowered incubation temperatures, the amount of labeled 20:4 and 20:5 in the EPG fraction was diminished; there was no analogous reduction of these labeled fatty acids in CPG.

\section{Discussion}

It is evident that culture at ambient temperatures below $37^{\circ}$ induces profound alterations in the lipid metabolism of dissociated brain cells.

At lowered incubation temperatures the formation of the higher polyunsaturated fatty acids of the $\omega 6$ and the $\omega 3$ series becomes inoperative. Although elongation and desaturation of $18: 2$ to $20: 3$ and desaturation of $20: 3$ to $20: 4 \omega 6$ (arachidonic acid) are temperature sensitive to an equal extent (Table II), the metabolism of 18:3 involves reactions possessing different temperature sensitivities. While the two chain elongation steps, the conversion of $18: 3$ to $20: 3$, and of $20: 5$ to $22: 5$, are significantly slowed by reducing the incubation temperature from $37^{\circ}$ to $30^{\circ}$, the desaturation reactions: $20: 3 \rightarrow 20: 4 \rightarrow 20: 5$ remain unimpaired until at least $15^{\circ}$ (Table IV).

As a consequence of the impairment of chain elongation the two major fatty acid components of the EPG fraction, 22:5 and 22:6, are not produced from 18:3, and incorporation into the $\mathrm{EPG}$ fraction of 18:3 and the other $\omega 3$ polyunsaturated fatty acids derived from 18:3 is markedly defective. From Table IV we can demonstrate that the ratio of labeled $20: 3+20: 4 / 22: 5+22: 6$ incorporated into the EPG fraction remains constant at all incubation temperatures, which suggests that fatty acid incorporation into the EPG fraction is a structurally specific process. No such specificity for the incorporation of labeled fatty

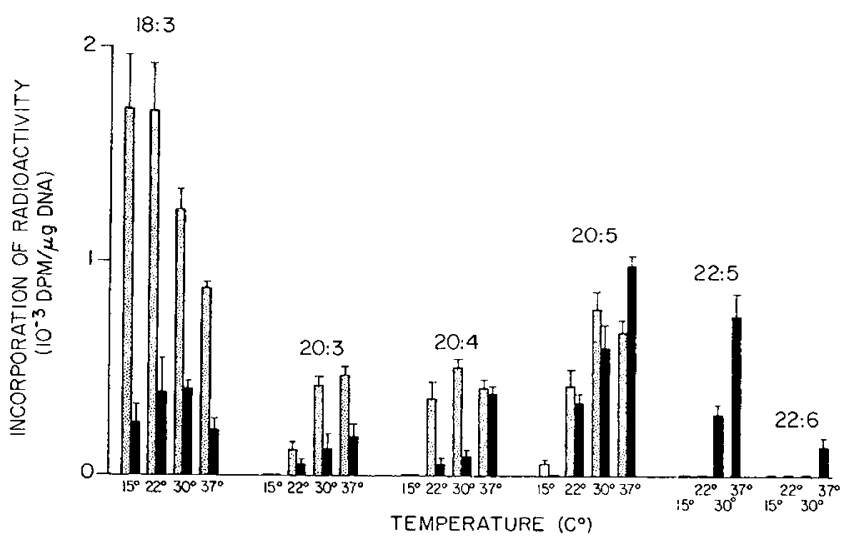

Fig. 3. Distribution of radioactivity from $\left(1-{ }^{14} \mathrm{C}\right)$ linolenic acid in fatty acid esters of choline phosphoglycerides (CPG) and ethanolamine phosphoglycerides and plasmalogens (EPG). Incubation conditions were the same as described for Table II. Analysis of fatty acid methyl esters of CPG (stippled bar) EPG (solid bar) was performed as described in Methods. Values expressed as disintegrations per minute per microgram of cellular DNA represent mean \pm SD of two analyses of three cultures. 
acids into CPG can be demonstrated. Because a large proportion of the EPG fraction is in the form of plasmalogens, a major myelin constituent, this specificity of fatty acid incorporation becomes understandable, as it may be necessary for the biosynthesis of myelin precursors.

There is considerable evidence that fatty acids from CPG are transferred to EPG [11]. The means by which this transfer is effected are still unknown, but it is likely that the fatty acids are cleaved from GPG to enter an intracellular fatty acid pool, where they are subject to chain elongation or desaturation, and subsequently are transferred to EPG.

The increased incorporation of 18:3 into CPG at lowered incubation temperatures (Fig. 3; Table III), suggests the presence of a temperature effect on fatty acid cleavage from CPG. The increased amounts of radioactivity in the fraction containing free fatty acids at lowered incubation temperatures (Tables I and III), is consistent with this hypothesis, and indicates that incorporation of free fatty acids into the EPG fraction and re-entry of free fatty acids into CPG may also become slowed when the incubation temperatures are lowered.

Our observations on the effects of ambient temperature on the synthesis of the higher polyunsaturated fatty acids, and on the formation of EPG, suggest that hypothermia may impair formation of membranes, possibly including myelin, in premature infants. Other factors, such as a significantly lowered cellular linoleic acid concentration, which has been demonstrated for erythrocytes of premature infants as compared with full term infants or adults [7], a high oxygen atmosphere, and vitamin $\mathrm{E}$ deficiency may contribute to the diminished availability of the essential fatty acid precursors for the higher polyunsaturated fatty acids required in the formation of cellular membranes within the developing nervous system.

\section{Summary}

Under lowered ambient temperatures, the conversion of linoleic acid (18:2) and linolenic acid (18:3) to the higher polyunsaturated fatty acids is impaired. Chain elongation of the essential fatty acids appears to be more sensitive to temperature reduction than their desaturation. Lacking 22:5 $\omega 3$ and 22:6 $\omega 3, \mathrm{EPG}$ and ethanolamine plasmalogen biosynthesis are severely curtailed, although incorporation of labeled fatty acids into the CPG fraction continues even at $15^{\circ}$. These alterations may result in defective deposition of membranes in hypothermic infants.

\section{References and Noles}

1. Bornstein, M. B., ANd Model, P. G.: Development of synapses and myelin in cultures of dissociated embryonic mouse spinal cord, medulla and cerebrum. Brain Res., 37: 287 (1972).

2. Burton, K. C., AND Klern, S. W.: Effect of maintenance of "normal" skin temperature on survival of infants of low birth weight. Pediatrics, 34: 163 (1964).

3. Day, R. L., Caliguiri, L., Kamenski, C., ANd Ehrlich, F.: Body temperature and survival of premature infants. Pediatrics, 34 : 171 (1964).

4. Glass, L., Silverman, W. A., and Sinclair, J. C.: Effect of the thermal environment on cold resistance and growth of small infants after the first week of life. Pediatrics, 41: 1033 (1968).

5. Jolly, H., Molyneux, P., ANd Newell, D. J.: A controlled study of the effect of temperature on premature babies. J. Pcdiat., 60: 889 (1962).

6. MANn, T. P., AND Ellrott, R. I. K.: Neonatal cold injury. Lancet, $i$ : 229 (1957).

7. Neerhout, R. G.: Erythrocyte lipids in infants with low birth weight. Pediat. Res., 5: 101 (1971).

8. Schatn, R. J., AND Watanabe, K.: Postnatal changes in protein metabolism of brain. II. Effects of alteration of ambient temperature and gaseous composition of inspired air. Pediat. Res., 5: 173 (1971).

9. SEEDS, N. W.: Biochemical differentiation in reaggregating brain cell culture. Proc. Nat. Acad. Sci. U. S. A., 68: 1858 (1971).

10. Sensenbrenner, M., Booher, J., And Mandel, P.: Cultivation and growth of dissociated neurons from chick embryo cerebral cortex in the presence of different substrates. Z. Zellforsch. Mikrosk. Anat., 117: 559 (197I).

11. SHowet, S. B.: The apparent transfer of fatty acid from phosphatidylcholine to phosphatidylethanolamine in human erythrocytes. J. Lipid Res., 12: 139 (1971).

12. Sulverman, W. A., Fertig, J. W., and Berger, A. P.: The influence of the thermal environment upon the survival of newly born premature infants. Pediatrics, $22: 876$ (1958).

13. VARON, S., AND RAiborn, C. W.: Dissociation, fractionation and culture of embryonic brain cells. Brain Res., 12: 180 (1969).

14. Yavin, E., AND Menkes, J. H.: The culture of dissociated cells from rat cerebral cortex. J. Cell Biol., 57: 232 (1973).

15. Yavin, E., ANd Menkes, J. H.: Glyceride metabolism in cultured cells dissociated from rat cerebral cortex. J. Neurochem. 21: 901 (1973).

16. YAVIN, E., AND MENKES, J. H.: The incorporation and metabolism of fatty acids by cultured dissociated cells from rat cerebrum. Lipids (in press).

17. Yavin, E., AND MEnKes, J. H.: Polyenoic acid metabolism in cultured dissociated brain cells. J. Lipid Res. (in press).

18. Grand Island Biochemical Co., Grand Island, N. Y. 
19. Rehatuin Reheis Chemical Corp., Chicago, III.

20. Amersham-Searle, Arlington Heights, Ill.

21. Merck A. G., Darmstadt, Germany.

22. Supelco, Bellefonte, Penna.

23. This research was supported by National Institutes of Health Research Grants nos. NS 06938 and CA 13538.
24. The authors gratefully acknowledge the outstanding assistance of Dr. Ziva Yavin, Mrs. Natalie Stein, and Mr. Donald Harris.

25. Requests for reprints should be addressed to: J. H. Menkes, M.D., Division of Pediatric Neurology, UCLA School of Medicine, Los Angeles, Calif. 90024 (USA).

26. Accepted for publication December 11, 1973. 